\title{
Successful robotic mitral valve plasty for isolated clefts of both anterior and posterior mitral valve leaflets
}

\author{
Norihiko Ishikawa ${ }^{1}$, Go Watanabe ${ }^{2}$, Kazuto Miyata ${ }^{2}$, Sayaka Shigematsu ${ }^{1}$, Takafumi \\ Horikawa $^{1}$, Ryuta Seguchi ${ }^{1}$, Ryuta Kiuchi ${ }^{1}$, Shigeyuki Tomita ${ }^{1}$, and Toshiya Ohtsuka ${ }^{1}$ \\ ${ }^{1}$ NewHeart Watanabe Institute \\ ${ }^{2}$ Affiliation not available
}

July 2, 2020

\begin{abstract}
Double clefts of both the anterior and posterior leaflets are extremely rare. A 21-year-old female with mitral valve regurgitation was admitted to our institute, and real-time three-dimensional transesophageal echocardiography revealed a cleft in the posterior mitral valve leaflet and an additional partial cleft in the anterior leaflet. Totally-endoscopic mitral valve plasty using surgical robot with direct suturing of both clefts, and annuloplasty were performed successfully. This is the first report of robotic totally-endoscopic mitral valve plasty for mitral regurgitation due to double clefts of the anterior and posterior mitral valve.
\end{abstract}

Echocardiography: Images

Successful robotic mitral valve plasty for isolated clefts of both anterior and posterior mitral valve leaflets

Running title: Robotic MV plasty for isolated clefts of MV

Norihiko Ishikawa1, Go Watanabe1, Kazuto Miyata2, Sayaka Shigematsu2, Takafumi Horikawa1, Ryuta Seguchi1, Ryuta Kiuchi1, Shigeyuki Tomita1, Toshiya Ohtsuka1

1. NewHeart Watanabe Institute, Department of Cardiovascular Surgery

2. NewHeart Watanabe Institute, Department of Anesthesia

Corresponding author

Norihiko Ishikawa, MD PhD

NewHeart Watanabe Institute

3-19-11 Hamadayama, Suginami, Tokyo 168-0065, Japan

Tel: +81-33311-1119, Fax: +81-33311-3119

E-mail: iskwnrhk@gmail.com

Keywords: robot, mitral valve, cleft, minimally invasive, mitral regurgitation

Abstract

Double clefts of both the anterior and posterior leaflets are extremely rare. A 21-year-old female with mitral valve regurgitation was admitted to our institute, and real-time three-dimensional transesophageal echocardiography revealed a cleft in the posterior mitral valve leaflet and an additional partial cleft in the anterior leaflet. Totally-endoscopic mitral valve plasty using surgical robot with direct suturing of both 
clefts, and annuloplasty were performed successfully. This is the first report of robotic totally-endoscopic mitral valve plasty for mitral regurgitation due to double clefts of the anterior and posterior mitral valve.

A 21-year-old female patient with a history of mitral valve regurgitation was admitted to our institute for surgery. She was being followed up at another hospital with a diagnosis of functional mitral regurgitation (MR) following detection of a heart murmur when she was in kindergarten.

Her functional capacity was NYHA class II. She was in sinus rhythm with a ventricular rate of $67 \mathrm{bpm}$. Transthoracic echocardiography revealed dilatation of left heart chambers with a left ventricular ejection fraction of $54 \%$, along with severe mitral regurgitation. Real-time three-dimensional transesophageal echocardiography (3D-TEE) revealed a cleft in the posterior mitral valve leaflet and an additional partial cleft in the anterior leaflet (Figure 1). After confirming the echocardiographic findings, we decided to perform robot-assisted minimally-invasive surgery.

Totally-endoscopic mitral valve plasty using the da Vinci surgical system (Intuitive Surgical, Inc., Sunnyvale, CA) with direct suturing of both the anterior and posterior clefts using expanded polytetrafluoroethylene sutures, and annuloplasty with a 29-mm annuloplasty band were performed (Figure 2). Operative time, cardiopulmonary bypass time and cross-clamp times were 225, 156 and 56 minutes, respectively. No blood transfusions were required and her postoperative course was uneventful.

\section{Comment}

To our knowledge, this is the first report of robotic totally-endoscopic mitral valve plasty for severe mitral regurgitation due to double clefts of the anterior and posterior mitral valve.

Not only are clefts of the posterior leaflet rarely reported, but double clefts of both the anterior and posterior leaflets are extremely rare and only a few such cases have been described so far ${ }^{1}$. The mechanism of development of congenital mitral valve clefts is not fully understood ${ }^{2}$, although it might be related to abnormal embryological development of endocardial cushion tissue.

Pre-operative 3D-TEE was very useful in identifying the clefts in the anterior and posterior mitral valve leaflets as an additional anatomic lesion, and was essential for planning the surgical procedure.

Surgical treatment options for mitral valve clefts with severe MR range from plasty to replacement ${ }^{2}$. Usually, direct suturing of the isolated cleft is preferred over mitral valve reconstruction ${ }^{3}$. However, if direct suturing is not feasible, mitral valve repair is preferable to valve replacement in adults. In our case, we opted for simple direct closure of both clefts with annuloplasty via small ports using a surgical robot.

\section{References}

1. Mohammadi S, Bergeron S, Voisine P, et al. Mitral valve cleft in both anterior and posterior leaflet: an extremely rare anomaly. Ann Thoracic Surg. 2006;82:2287-2289.

2. Du X, Hung C, Wan Z. Successful mitral valve repair for severe mitral regurgitation caused by isolated cleft of the posterior leaflet. Int J Cardiol. 2011;150:48-49.

3. Perier P, Clausnizer B. Isolated cleft mitral valve: valve reconstruction techniques. Ann Thorac Surg. 1995; 59: 56-59.

Figure legends

Figure 1. Real-time three-dimensional transesophageal echocardiography image showing clefts in both the anterior and posterior mitral valve leaflets

Figure 2. Intraoperative view of the mitral valve from the left atrium

A: Clefts of the anterior mitral leaflet (white arrow) and the posterior mitral leaflet (black arrow) are visible.

B: Robotic direct closure of the posterior cleft was performed. 
C: Robotic direct closure of the anterior cleft was performed.

D: Annuloplasty was performed using an annuloplasty band.
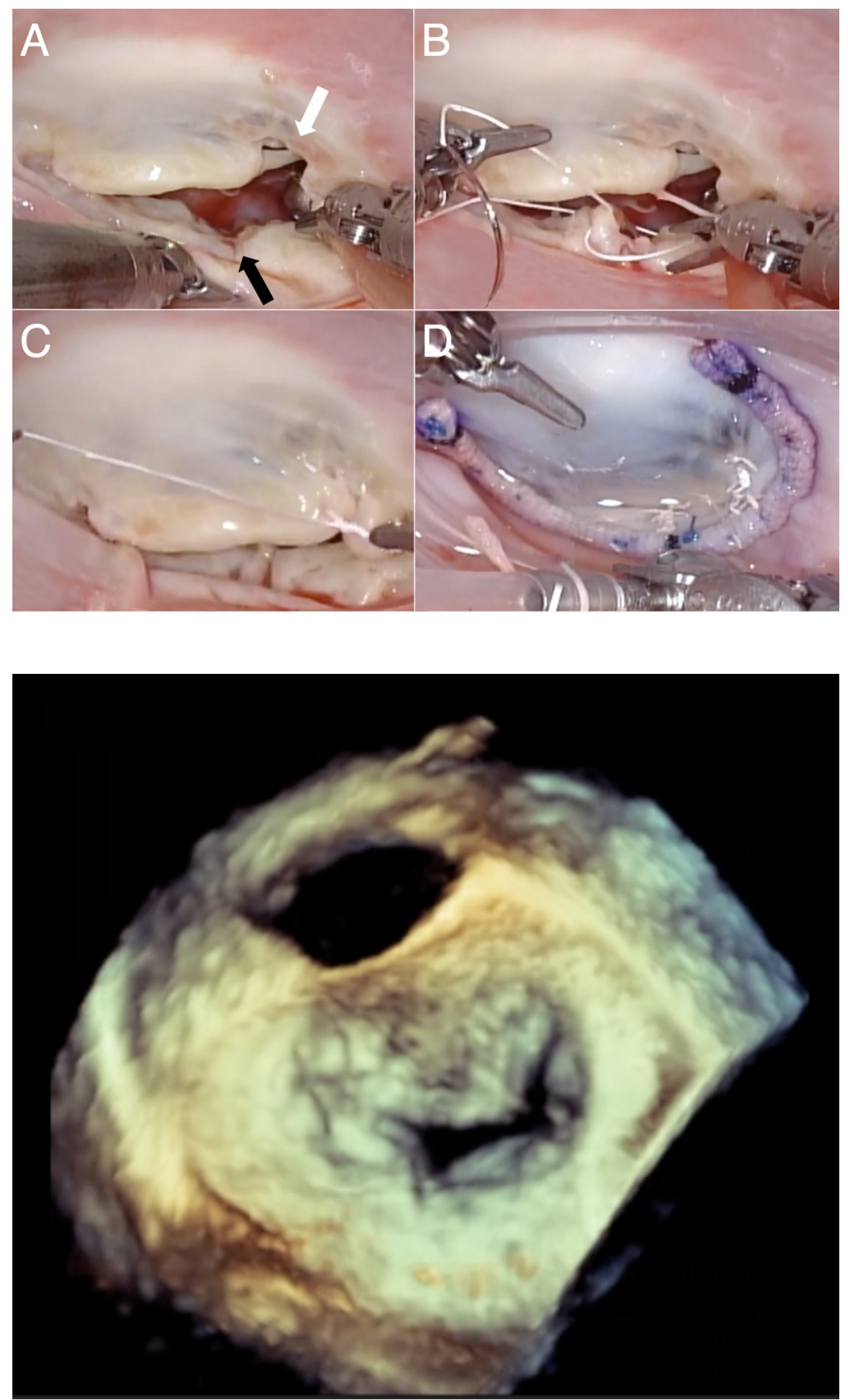


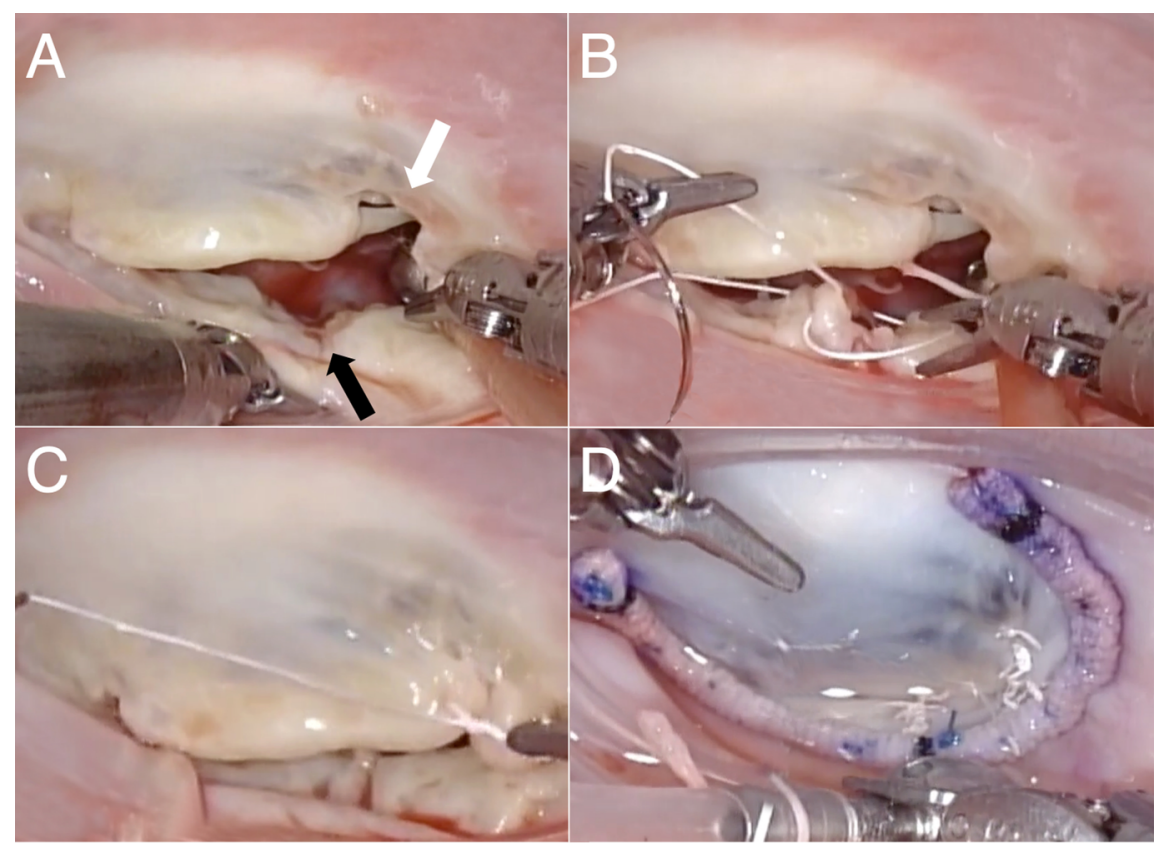

